\title{
Las Mesas de Gestión de los Centros Integradores Comunitarios: ¿participación de la comunidad y democratización de las políticas sociales? *1
}

\author{
The Tables of Management of Community Integrating Centers: community \\ participation and democratization of social policies?
}

\author{
Leonel Del Prado**
}

\section{Resumen}

En el presente artículo se reflexionó sobre un dispositivo de participación promovido por el Estado en el marco de una política pública del Ministerio de Desarrollo Social de la Nación Argentina, denominada Mesa de Gestión. Dichas mesas forman parte de los Centros Integradores Comunitarios (CIC), 812 edificios en los que se realizan intervenciones vinculadas al desarrollo social y la prevención primaria de la salud, los que se construyeron desde 2004 al 2015 durante los gobiernos kirchneristas. Se afirma en este trabajo que la existencia de dicha metodología participativa no necesariamente aporta a la participación de la comunidad y la democratización de las políticas sociales. Se tomó como punto de análisis una observación participante realizada en el $\mathrm{CIC}$ de la ciudad de Concordia entre 2010 y 2011 -Entre Ríos, Argentina- y los trabajos finales de especialistas que llevaron adelante sus intervenciones en diferentes $\mathrm{ClC}$ del país, en el marco de un posgrado financiado por el Ministerio de Desarrollo Social de la Nación.

\section{Abstract}

The article herein presents a participation device promoted by the State in the frame of a social development policy, called

\footnotetext{
* Artículo recibido 23 de septiembre de 2016. Aceptado 27 de febrero de 2017.

** Licenciado en Trabajo Social (UNER) - Estudiante del Doctorado en Ciencias Sociales (UBA). Docente investigador en la Universidad Autónoma de Entre Ríos. Correo electrónico: leonelts@hotmail.com
} 
Mesa de Gestión, of the Argentinian Ministry of Social Development (Ministerio de Desarrollo Social de la Nación Argentina). It is integrated by Community Integration Centres (CICs, Centros Integradores Comunitarios), 812 buildings, built in 2004 and 2015 during Kirchner's governments, which make interventions linked to social development and health primary prevention.

It was stated that the existence of those inclusive methodologies not necessarily contribute to the participation of the community and nor to the democratization of the social policies, but they consolidate the disparities of the politic field. The analysis focus was a participative observation that took place in $\mathrm{CIC}$ from Concordia city (Entre Ríos, Argentina) between 2010 and 2011 and the specialists' final works that took place in different $\mathrm{ClCs}$ of the country in a postgraduate framework funded by the Argentinian Ministry of Social Development.

\section{Palabras clave}

Participación - Mesas de Gestión - Centros Integradores Comunitarios.

\section{Keywords}

Participation - Management Table - Community Integration Centres.

\section{Introducción}

Las políticas del Ministerio de Desarrollo Social de la Nación Argentina -MDSNimplementadas durante los tres gobiernos kirchneristas, bajo la dirección de la doctora Alicia Kirchner (Del Prado, 2015), construyeron a lo largo de los años un discurso de las políticas sociales: las nuevas políticas sociales, asociadas a un cambio en la intervención del Estado en materia social.

En ese marco, en las alocuciones de la ministra, en diferentes publicaciones, y en el trabajo cotidiano de los profesionales del MDSN en el territorio, se planteaba la existencia de dichas nuevas políticas sociales a partir de 2003, año en que asumió la Presidencia de la Nación Néstor Carlos Kirchner. Discursivamente, las nuevas políticas sociales se constituyeron en oposición a las políticas sociales neoliberales, planteando la dicotomía de un Estado ausente neoliberal ${ }^{2}$ contra un Estado "presente, activo, protagónico" del gobierno kirchnerista (Kirchner, 2007a:15).

\footnotetext{
${ }^{2}$ Existe un conjunto de autores que desde perspectivas diferentes van a cuestionar el tema del "Estado mínimo" del neoliberalismo: Morresi afirma que ninguna de las diferentes escuelas neoliberales promueve un Estado mínimo, sino que todas le otorgan un rol importante (2007:127); Oszlak, por su parte, afirma que "considerar que se está en presencia de un Estado "mínimo" por el hecho de que el gobierno nacional se
} 
En la perspectiva de este discurso esgrimido desde el Ministerio, un conjunto de intelectuales en el campo de las ciencias sociales de Argentina plantea que se gestaron transformaciones en el marco de las políticas sociales (Martínez, 2015; Castronovo y García, 2013; Meroni, 2013; Vilas, 2011; Bazzalo, 2011; Arroyo, 2009; Repetto y Chudnovsky, 2009; García Delgado, 2006) y específicamente con relación a los CIC refieren que los mismos se constituyen en mecanismos orientados a fomentar la participación comunitaria en las políticas sociales (Castronovo y García, 2013; Meroni, 2013; De Piero, 2015).

El tema de la participación bajo los gobiernos kirchneristas ha sido ambivalente, en tanto, si bien se proponía la democratización vinculada a la participación de los beneficiarios de las políticas sociales, el período de creación de la política de los CIC año 2004- fue un período en el cual hubo una fuerte concentración de las decisiones en torno al presidente y poco lugar al parlamento y/o foros ciudadanos (Sidicaro, 2011:268-269).

Si bien se plantea que las nuevas políticas sociales poseen entre sus ejes centrales la democracia y la participación; según la investigación desarrollada por el abogado Carrasco (2012), en las instancias formales de discusión de un país republicano como el argentino, es decir el Congreso nacional, no se encontraron presentes, ya que las distintas propuestas de políticas sociales fueron presentaciones realizadas por el Ejecutivo nacional, las que no solo no recibieron modificaciones, sino que en muchos casos ni siquiera se discutieron y fueron aprobadas de forma automática, dado el poder que poseía el kirchnerismo en los primeros años de gobierno en ambas cámaras (Carrasco, 2012).

En ese contexto de debates es que se reflexiona sobre este dispositivo de participación promovido por el Estado en el marco de una política de desarrollo social del Ministerio de Desarrollo Social de la Nación Argentina: las Mesas de Gestión de los Centros Integradores Comunitarios.

Durante la investigación se concibió al Estado no solo como productor de representaciones y constructor de la realidad (Bourdieu, 1997), sino también al mismo, sus agentes y sus políticas como un producto cultural (Corrigan y Sayer, 2007; Corrigan, 2002); con el objetivo de comprender e interpretar la política, se realizó un trabajo de campo entre 2010 y 2011 desde una metodología de tipo cualitativa: entrevistas abiertas, observación participante de diversas situaciones y eventos tomando como ámbito de observación el Centro Integrador Comunitario de la ciudad de Concordia, provincia de Entre Ríos, Argentina.

Para el presente trabajo se toman las observaciones realizadas en las reuniones de la mesa de gestión, el libro de actas de dichas reuniones, documentos oficiales referidos 
al CIC y los trabajos finales de especialistas que se llevaron adelante en diferentes CIC del país, en el marco de la Especialización en Abordaje Integral de las Problemáticas Sociales en el Ámbito Comunitario, posgrado realizado en la Universidad de Lanús y financiado por el Ministerio de Desarrollo Social de la Nación, esto último se hace con el objetivo de comparar la experiencia de la ciudad de Concordia con otras llevadas adelante en Argentina.

En el artículo se desarrolla la política de los Centros Integradores Comunitarios y luego se pone énfasis en el dispositivo de la Mesa de Gestión, ya que posteriormente se tensiona el caso de la ciudad de Concordia con distintas experiencias en diferentes puntos del país. Finaliza el trabajo con unas reflexiones en torno a las Mesas de Gestión y las prácticas participativas en el marco de dispositivos estatales.

\section{Los Centros Integradores Comunitarios}

Las políticas de desarrollo social kirchneristas se plantean como políticas que no solo no son universales (es decir, las del Estado de Bienestar) ni focalizadas (las del Estado Neoliberal), sino que se da cuenta que esta discusión está superada por las "políticas integrales" (Kirchner, 2007a y 2010a). Según el discurso oficial esgrimido desde el MDSN, el problema de las políticas sociales de los períodos previos permanecería en la forma en cómo estas se implementan, la integración de las respuestas del Estado o "la integralidad", que vendrían a superar este problema.

Una de las políticas del Ministerio que da cuenta de lo que en el discurso oficial se denomina cambio de paradigma son los Centros Integradores Comunitarios, una de las políticas eminentemente kirchneristas, ya que su protoforma fue desarrollada en la provincia de Santa Cruz.

Kirchner y Vessvessian, en una publicación de $2001^{3}$, la definen como el "Modelo santacruceño de abordaje" de la realidad social (2001: 71), dicha política con la llegada de Néstor Kirchner a la Presidencia de la Nación fue replicada a lo largo y a lo ancho del país, llegando al final de los tres períodos a un total de 812 CIC construidos (MOST, 2015).

La Resolución 2173 del Ministerio de Desarrollo Social de la Nación Argentina con fecha del 19 de julio de 2004, firmada por los titulares de los Ministerios de Salud; Trabajo, Empleo y Seguridad Social; Planificación Federal e Inversión Pública; Desarrollo Social; y del Consejo Nacional de Coordinación de Políticas Sociales, fue el puntapié

\footnotetext{
${ }^{3}$ El libro En Busca del Ordenador Social. Trabajo: agenda no resuelta es fruto de una reflexión sobre la gestión que llevaron adelante en Santa Cruz. El texto hace énfasis en los denominados "modelos asociativos" que buscan la solución a las necesidades mediante una articulación entre el Estado, el sector privado y las organizaciones sociales. Las autoras plantean construir una "red de servicios de empleos localizados en las necesidades, potenciados en nuestra propuesta a partir de los Centros Integradores Comunitarios, desde una gestión a tres hilos: público, privado y del tercer sector" (Kirchner y Vessvessian, 2001: 15 y 16).
} 
para llevar adelante una política de desarrollo social denominada Centros Integradores Comunitarios. Para implementar dicho proyecto en el territorio nacional se construyeron edificios denominados $\mathrm{CIC}$, los cuales tienen como finalidad brindar políticas de atención primaria de la salud y de desarrollo social. Dichas instituciones se encuentran ubicadas en "los territorios más vulnerables del país" (Kirchner, 2008: 142) ${ }^{4}$. En el libro del Bicentenario (Kirchner, 2010) se la define como:

Son el Estado presente en el territorio. Puertas abiertas hacia y en la comunidad, no encerradas en una visión puramente institucionalizada. Su espacio es promovido por el Estado para ser construido y habitado por y con la comunidad. Así, se anclan en la sociedad civil, en tanto que desde una perspectiva territorial potencian la vida democrática. Tienen como eje la activación de los recursos, las potencialidades y el pleno desarrollo de las capacidades de cada comunidad (Kirchner, 2010:105).

Nombrado de diferentes formas, el CIC remite a una forma de gestión, una práctica ejemplar para cumplir con los Objetivos de Desarrollo del Milenio, como una herramienta del territorio (Kirchner, 2010), es decir que desde el discurso oficial se trató permanentemente de mostrarlos y construirlos como mucho más que edificios que brindan prestaciones de salud y desarrollo social.

\section{Las Mesas de Gestión}

Los CIC como organizaciones forman parte de las políticas del Ministerio de Desarrollo Social de la Nación, las cuales refieren permanentemente a la participación de la comunidad en la gestión de las políticas sociales y refieren a los destinatarios de las políticas sociales como protagonistas.

En este caso, las Mesas de Gestión o Mesas de Gestión Local se constituyen como una metodología participativa que rige el $\mathrm{CIC}$ y se encuentra conformada por diferentes actores comunitarios; esta modalidad de trabajo fue creada junto al asesor internacional en políticas sociales, Bernardo Kliksberg ${ }^{5}$, y había sido trabajada con anterioridad en los Consejos Consultivos del Plan Jefes y Jefas de Hogar Desocupados ${ }^{6}$ (Kirchner, 2008:108).

\footnotetext{
${ }^{4}$ Para mayor información sobre los CIC, ver Kirchner 2007a, 2007b, 2008 y 2010a.

5 Economista argentino vinculado a temas de pobreza, gerencia social y responsabilidad social empresarial. Se desempeña como Asesor Principal de la Dirección Regional para América Latina y el Caribe del Programa de Naciones Unidas para el Desarrollo (PNUD) y como Director del Fondo España-PNUD "Hacia un desarrollo integrado e inclusivo en América Latina y el Caribe", instituciones con las cuales ha realizado numerosos foros, encuentros y publicaciones. Ha sido asesor de diversos países y organismos internacionales como la ONU, UNESCO, UNICEF, OIT, OEA, OPS. Estudió en la Universidad de Buenos Aires, institución que lo nombró Gran Maestro. Ha escrito más de 40 libros, entre los que se destaca el escrito con Amartya Sen, Primero la Gente.

${ }^{6}$ El "Plan Jefes y Jefas de Hogar Desocupados" se implementó en el año 2002, en un intento del entonces presidente Eduardo Duhalde de dar respuesta a la crisis de 2001, contó con el apoyo de la Iglesia católica y
} 
El diseño de la política de los CIC busca que las personas reunidas en la Mesa de Gestión establezcan cuáles son sus problemas y cuáles son las soluciones a los mismos, esta visión persigue la finalidad de atacar uno de los problemas básicos de las políticas sociales, es decir que las mismas sean pensadas por agentes externos a la situación problemática -asesores internacionales, profesionales, políticos-. Este enfoque se conoce como planificación estratégica ${ }^{7}$ y ha sido vulgarmente conocido como planificación de "abajo hacia arriba", dentro de la tecnología de la planificación se ubicaría al interior de aquellas formas de planificación alternativas a la tradicional, de vertiente economicista.

En los diferentes documentos oficiales se planteaba que los Centros Integradores Comunitarios eran llevados adelante por distintas instancias gubernamentales -la Nación, las provincias y los municipios- y la comunidad en el marco de la Mesa de Gestión, espacio en el cual, según los textos oficiales, se discuten, planifican y evalúan las políticas sociales. Esto fue constantemente remarcado de modo prescriptivo en diferentes espacios: la comunidad no puede ser mera receptora de programas. Autoridades del Ministerio y profesionales recalcaban de manera permanente la transformación que se generó en las políticas sociales, en palabras de Alicia Kirchner: "el gran cambio que ponemos, la bisagra que existe en la historia de la política social, basada en la participación, donde también se promueve y se asiste, porque donde hay una necesidad hay un derecho, pero fundamentalmente se trabaja sobre la participación que es lo que dignifica" (Kirchner, 2007a:305).

Como uno de los logros de la política de los Centros Integradores Comunitarios, se planteó la existencia de las Mesas de Gestión, es algo que la ministra Alicia Kirchner remarcaba en cada una de sus exposiciones, dado que las mismas forman lo que en el discurso oficial se denominó la democratización de las políticas sociales; las mesas de gestión eran definidas como:

espacios abiertos y participativos, a los cuales todos los vecinos pueden acercarse a presentar, evaluar y priorizar propuestas de lo que queremos y necesitamos hacer (...) Son espacios donde se discute, se debate y se acuerdan posibles soluciones colectivas, en donde se asumen compromisos, responsabilidades y se distribuyen tareas... (Kirchner, 2010b: 66).

En el texto compilado por Bernardo Kliksberg, Pensamiento Social Estratégico. Una nueva mirada a los desafíos sociales de América Latina, la ministra Kirchner realizó una exposición denominada "Los Centros Integradores Comunitarios. Sentido de la Política

el Programa de las Naciones Unidas para el Desarrollo -PNUD-. Entre las características que sobresalen del programa se destaca su masividad, alrededor de dos millones de beneficiarios. Daniel Arroyo afirma que dicho plan "universalizó la construcción de los espacios asociativos" entre el Estado, las organizaciones sociales y los privados, los que a fines del año 2002 ascendían a 1754 Consejos Consultivos Locales (2004:13). Para un análisis detallado de dicho plan, ver Golbert (2004).

${ }^{7}$ Los autores que se toman como referencia desde esta perspectiva son Carlos Matus (1972) y Mario Robirosa, Graciela Cardarelli y Antonio La Palma (1990). 
Social=El Umbral del Cambio". La misma se presenta en el texto como una experiencia ejemplar. En él la ministra remarcó: "Cada comunidad en particular elige lo que necesita para su centro", y se remarca la importancia de los CIC y de las mesas de gestión como un "motor del desarrollo" (Kirchner, 2008: 110).

En el texto del Bicentenario (Kirchner, 2010a) se realiza un cuadro con los Sí y los No de las políticas sociales, en las que se establece un conjunto de características de las políticas en el marco del Proyecto Nacional y Popular contrapuesto a las características del Modelo Neoliberal; en las primeras se establece:

Desarrollo de 1100 mesas de gestión y participación: comprende la organización social plural. Todas las personas deben construir su destino; el Estado debe promover las oportunidades (...) El CIC es la herramienta no sólo de prestación de servicios sociales y de salud, sino de organización (vecinales, sociedades de fomento, centro de jubilados, entre otros) ... (Kirchner, 2010a:276).

En contraposición a ello, refiere al "Desarrollo del onegeismo: reservado a 'expertos' del sector social" (ídem), el cual se había desarrollado en tiempos previos al gobierno de Kirchner, período denominado como neoliberal. Esta dicotomía "onegeísmo" versus "desarrollo de mesas de gestión y participación" se suma a las concepciones binarias, dicotómicas y maniqueas del discurso de las nuevas políticas sociales.

No existía ninguna norma respecto de cómo debía ser la Mesa de Gestión, cómo debía ser conformada y cuáles eran sus características; ello perseguía la finalidad de respetar las formas de organización de cada lugar en el cual se encontraba inserto el CIC (Del Prado, 2009: 65). Cuando comenzó el proyecto CIC, la idea desde el Ministerio era que fuesen llevados adelante por las Mesas de Gestión, pero esto encontró resistencias en los municipios y en las provincias. Finalmente, los CIC contarían con su Mesa, pero el municipio en donde se asentaba el CIC pondría al coordinador de la organización, el que a su vez podía ser el coordinador de la mesa ${ }^{8}$.

En el cuadernillo del Consejo Nacional de Coordinación de Políticas Sociales que se distribuyó en los diferentes $\mathrm{CIC}$ denominado CIC. Centro Integrador Comunitario. Una oportunidad para la organización, Alicia Kirchner, luego de hacer un repaso por la historia y la nueva concepción de las políticas sociales, aborda de lleno los CIC, y dentro de sus características remarca la noción de la Mesa de Gestión Local, pero no se establece una definición, sino que se recuperan distintos relatos de vecinos que narran lo que realizan y cómo trabajan en dicho dispositivo de participación (2007b: 31). En dichos relatos se recupera la posibilidad del encuentro, el debate, la discusión, el voto, asociando dicho espacio con la participación, la organización y la democratización de las políticas sociales.

\footnotetext{
${ }^{8}$ Como estudiante de la Especialización de Abordaje Integral de Problemáticas Sociales en el Ámbito Comunitario de la primera cohorte (2006 a 2008), viví en primera persona estos inconvenientes en el CIC de la ciudad de Gualeguay.
} 
En los siguientes puntos se describe cómo funciona el $\mathrm{ClC}$ de Concordia y su mesa; y posteriormente otras experiencias en el país desde el punto de vista de profesionales en el marco del posgrado.

\section{La Mesa de Gestión de Concordia: el coordinador, los referentes comunitarios y la comunidad}

Diferentes investigaciones sociales referidas a la ciudad de San Antonio de Padua de la Concordia dan cuenta de las distintas problemáticas que posee, entre las que se destacan: la pobreza, el desempleo estructural y la inequidad (Mingo et. al., 2006; Reta, Rossi y Toler, 2008). Otras características de la ciudad que dan cuenta de su particularidad son: ciudad intermedia - de 50 mil a 1 millón de habitantes-, ciudades que articulan el territorio, binucleares -Concordia y Salto-, concentración de equipamientos y recursos en la planta urbana, cinturón de villas en periferia y zonas inundables, importancia de la represa de Salto Grande (Mingo de Bevilacqua et. al., 2006: 78-79; País Andrade, 2010).

Para abordar las distintas problemáticas en un barrio de la zona noroeste de la ciudad de Concordia, en 2007 se inauguró un CIC, el cual se encuentra en funcionamiento, prestando servicios de salud y desarrollo social en el barrio Sarmiento y su área de influencia.

Desde dicho año, la Mesa de Gestión del CIC de Concordia se reunía semanalmente con el objetivo de tratar diferentes problemáticas; como no estaba predefinido desde el Ministerio quiénes formaban parte de la Mesa, dado que el proyecto del CIC supone respetar las formas de organización locales; en el año en que se realizó el trabajo de campo -2011- la Mesa de Gestión estaba conformada de la siguiente manera: participan personas de diferentes instituciones del área de influencia ${ }^{9}$ del $\mathrm{CIC}$, para ello tienen que tener un aval de las organizaciones o grupos de los cuales forman parte, que diga que son ellos los que van a participar de la mesa. Los integrantes de la mesa son dichas personas ${ }^{10}$, y son los únicos que tienen voz y voto al interior de la Mesa de Gestión; a su vez, son los únicos que tienen el acceso al libro de actas del $\mathrm{CIC}$, dado que el 13 de enero de 2010 se decidió que "el libro de actas queda en la oficina del Sr.

\footnotetext{
${ }^{9}$ Se le denomina área de influencia o área programática a la zona que abarca el $\mathrm{CIC}$, los barrios lindantes.

10 Jardín Maternal, Área Salud, Educación, Talleres Culturales, Área Discapacidad, Cooperativas, Federación de Tierra y Vivienda -FTV-, Promotores Territoriales, Comisión Vecinal, Asociación Civil La Gloriosa, Cooperadoras de Padres. Como se puede ver, los cuatro primeros están vinculados a "sectores" del $\mathrm{CIC}$; las cooperativas son las que construyeron el edificio del CIC y se las convocó desde un primer momento a la Mesa de Gestión -en el año 2011 formaban parte del programa Argentina Trabaja-; FTV y La Gloriosa son organizaciones políticas vinculadas al kirchnerismo; la Comisión Vecinal -en la ciudad de Concordia las comisiones vecinales se encuentran regladas por la Dirección de Organizaciones Sociales y No Gubernamentales, perteneciente a la Secretaría de Gestión Participativa para el Desarrollo Local- y la Cooperadora de Padres de la escuela del barrio Juana Azurduy.
} 
-coordinador- y los únicos que tienen acceso a dicho libro son los integrantes de la mesa de gestión" -Libros de Actas, marzo de 2010-.

A las distintas reuniones muchas veces se acercaban vecinos con propuestas de talleres o cursos para dictar en el $\mathrm{CIC}$, lo único que realizaba la Mesa de Gestión en ese tipo de caso era escuchar el proyecto, debatirlo, y si se lo aceptaba, se procedía a darle el aval de la Mesa de Gestión, pero se le pedía a la persona que gestione los recursos para llevar adelante la propuesta, la mesa no disponía de fondos para el desarrollo de las actividades. El no tener recursos era visto como un obstáculo por los miembros de la mesa, dado que la existencia de fondos les permitiría, según su perspectiva, realizar más actividades. La provincia no tenía mucha injerencia en lo que respecta al $\mathrm{CIC}$, pero los funcionarios provinciales vinculados al proyecto $\mathrm{CIC}$ también veían como problemático esto y en un encuentro provincial de Mesas de Gestión desarrollado en la ciudad de Concepción del Uruguay propusieron armar cooperadoras en cada uno de los $\mathrm{ClC}$, apelando a la tradición de los inmigrantes en la provincia de Entre Ríos, pero esta propuesta no tuvo el aval de los profesionales del MDSN y quedó solo en una idea-proyecto.

Según lo que consta en el libro de actas, las reuniones de la Mesa de Gestión del CIC se realizaban desde el 5 de marzo de 2007, con periodicidad semanal. De la lectura y reflexión de las 200 páginas del libro de actas se pueden ver diferentes elementos comunes, entre ellos se destacan: a) la permanente conformación y reconfiguración de los asistentes a las reuniones de la Mesa de Gestión, y a su vez una preeminencia en la conformación de la mesa de personas vinculadas al Estado; b) los problemas a los que se enfrentaba la Mesa: la puja permanente con las autoridades del Municipio por la camioneta utilitaria del CIC -la cual era utilizada en otras dependencias-, y el pedido de refacciones y mejoras edilicias del $\mathrm{CIC}$; c) también sobresale en las actas, el pedido de diferentes instituciones, organizaciones y grupos del barrio para utilizar distintos espacios del CIC.

Si bien las distintas publicaciones realizadas por el MDSN referían a que la Mesa de Gestión es la que llevaba adelante el $\mathrm{CIC}$, en el caso de la ciudad de Concordia las decisiones sobre el funcionamiento cotidiano de la organización estaban en manos del coordinador del $\mathrm{CIC}$, él era un funcionario de la Municipalidad de Concordia y se encontraba desempeñando dicho cargo desde la apertura del $\mathrm{CIC}$; en una de las entrevistas refirió a que si bien el CIC era de Nación, él nunca podía patear en contra del Municipio ${ }^{11}$, porque el Intendente era su jefe, y él pertenecía a la Municipalidad. El coordinador es quien convocaba a las reuniones de la Mesa de Gestión, iniciaba las reuniones narrando las últimas noticias de la organización, es quien poseía el libro de actas en su oficina y era el representante del $\mathrm{CIC}$ en las diferentes actividades que se

\footnotetext{
${ }^{11}$ El coordinador es un trabajador del mundo del fútbol, por lo que muchas de sus reflexiones las expresa con metáforas deportivas, como en este caso "patear en contra".
} 
realizaban fuera de la organización, y en el cuaderno de actas se refiere que él es quien posee la representación legal del CIC. La voz del coordinador es la que predominaba en la institución y en las reuniones de la mesa, muchas veces en disputa con otros actores, los referentes comunitarios.

Los vecinos o la comunidad no participaban de la Mesa de Gestión como plantean los documentos oficiales, sino que son los representantes de la comunidad -es decir quienes representan a alguna organización o institución- los que participan en la mesa como miembros -con voz y voto- y en el día a día del CIC. Al observar el interior de la institución, entre dichos representantes ocupaban un lugar fundamental los referentes comunitarios o referentes. Estos poseían las características de habitar en el territorio del $\mathrm{CIC}$-es decir el área de influencia del mismo-, ser beneficiarios de las políticas del MDSN y militantes de agrupaciones vinculadas al kirchnerismo. Estos actores constituían su rol en el marco de las políticas de desarrollo social y se constituyeron en un actor fundamental, que era invocado por todos, aunque en el discurso oficial de las nuevas políticas sociales se ocultaba su rol, ya que estos actores eran cotidianamente convocados a trabajar, pero luego su accionar se diluía en la noción ambigua de comunidad.

Los referentes comunitarios eran personas de referencia de los profesionales del Ministerio nacional, con quienes se comunicaban, se reunían y mantenían un intercambio fluido; de las autoridades del CIC y el Municipio, quienes los convocaban a las diferentes actividades; de los vecinos, que acudían con pedidos o problemáticas; y ellos se concebían de ese modo, el cual era reconocido colectivamente, principalmente por su trabajo desinteresado por la comunidad; este reconocimiento es un capital simbólico $^{12}$ que era disputado cotidianamente entre los dos referentes comunitarios del $\mathrm{CIC}$ de Concordia y con otros actores que no habían logrado constituirse como referentes.

Estos referentes eran, a su vez, un objeto privilegiado por los profesionales del MDSN en la difusión del proyecto del $\mathrm{ClC}$, es por ello que eran invitados a encuentros, jornadas, talleres de todo tipo, en los que les entregaban folletos, libros e información, ya que muchas veces en la disputa por la construcción de lo que era un CIC entre la Nación, las provincias y los municipios, desde el Ministerio se los entendía como los guardianes del proyecto $\mathrm{CIC}^{13}$.

\footnotetext{
${ }^{12}$ Bourdieu distingue diferentes tipos de capital: económico, cultural y social, a los cuales a su vez agrega un cuarto tipo, el simbólico, que lo define como: "la forma que una u otra de estas especies adopta cuando se la entiende a través de categorías de percepción que reconocen su lógica específica o, si lo prefieren, desconocen la arbitrariedad de su posesión y acumulación" (Bourdieu y Wacquant, 2008:158-159). Alicia Gutiérrez, en su trabajo sobre Bourdieu, refiere a que el mismo es un "sobreañadido de prestigio, legitimidad, autoridad, reconocimiento, a los otros capitales..." (Gutiérrez, 1994:29).

${ }^{13}$ En muchos municipios de la Argentina, los edificios de los CIC fueron recreados como Centros de Salud o extensiones de los municipios; así como los vehículos utilitarios destinados a los CIC por parte del MDSN fueron ocupados por otras dependencias municipales, conflictos de este tipo constaté en mi rol de
} 
Este contacto personalizado entre profesionales de Nación y los referentes comunitarios, asimismo, es reconocido por todos los actores, y dichos referentes constituyen su identidad con la Nación, ya que en diferentes oportunidades se ubicaron como parte de la misma refiriendo: "Nosotros desde Nación". Esta identificación, a su vez, la realizan para distanciarse del Municipio y la Provincia, quienes serían los que en múltiples ocasiones quieren desvirtuar el proyecto de los CIC en beneficio personal, o acotándolo a un hospitalito o una extensión del municipio.

En cuanto a roles y predominio al interior del CIC y la mesa, predomina la voz del coordinador, los referentes comunitarios, el resto de los miembros de la Mesa y finalmente los vecinos o la comunidad, que se acercaban a las reuniones.

Finalmente, es importante remarcar que de las reuniones de la Mesa de Gestión participaban actores estatales y representantes de la comunidad que poseían un vínculo ideológico directo con el kirchnerismo, ya que si bien el mismo es heterogéneo en su interior, no hay actores que se encuentren por fuera del mismo o que sean opositores al grupo político que ocupó el Ejecutivo nacional entre 2003 y 2015, denominado por los diferentes actores como el Proyecto Nacional y Popular; las reuniones no constituían el espacio abierto y plural propugnado en los documentos oficiales, sino que había un acuerdo implícito de quienes eran los que podían participar.

\section{Otras experiencias de Mesas de Gestión: la voz de los especialistas El posgrado}

Desde el Ministerio de Desarrollo Social de la Nación se financió un conjunto de posgrados, el cual buscó capacitar a los profesionales en el marco de la implementación de "las nuevas políticas sociales", dichos posgrados estaban vinculados a diferentes temas: el Abordaje de Problemas Sociales en el Ámbito Comunitario; la Gerontología; la Niñez y la Juventud; la Salud Comunitaria -este último junto al Ministerio de Salud-; los mismos se realizaron en Universidades Nacionales Públicas. Con el objetivo que los profesionales en el marco del posgrado desarrollasen su práctica en los $\mathrm{CIC}$, la "Especialización en Abordaje Integral de Problemáticas Sociales en el Ámbito Comunitario", implementada por la Universidad Nacional de Lanús, desarrolló cinco cohortes entre 2006 y 2015 con 282 graduados.

El grupo que planificó la especialización plantea en un texto que reflexiona sobre la experiencia (Castronovo y García, 2013:31) que el desempeño de los profesionales se dio en el marco de un cambio de paradigma, el cual consistía en un nuevo tipo de desarrollo, un desarrollo integral e inclusivo. A dichas políticas, fruto del cambio que se gestó a partir del gobierno de Néstor Kirchner, las autoras lo denominan como políticas

estudiante de la Especialización en Abordaje Integral de Problemáticas Sociales en el Ámbito Comunitario en el CIC de la ciudad de Gualeguay (Entre Ríos) y durante la investigación desarrollada entre 2010 y 2011 en la ciudad de Concordia. 
sociales de nuevo cuño (Castronovo y García, 2013:13), en consonancia con la idea de las nuevas políticas sociales esgrimida desde el Ministerio de Desarrollo Social.

La Especialización poseía una modalidad denominada "modelo de estudio-trabajo en servicio"; es decir que la característica que distinguía a dicha carrera de otros posgrados era la denominada "Práctica en Servicio", la cual consistía en participar de las distintas actividades que desarrollaba el $\mathrm{ClC}$ al cual cada profesional había sido asignado, un profesional por $\mathrm{CIC}$, con el objetivo de realizar tareas semanalmente. Las mismas estaban pautadas en el diseño del posgrado y tenían el objetivo de tensionar los aprendizajes teórico-metodológicos de los distintos seminarios con la implementación de las políticas.

El posgrado duraba dos años, sumando en su totalidad 736 horas, de las cuales 240 correspondían al trabajo en servicio. Las diferentes materias dictadas fueron: Planificación Estratégica; Epidemiología Social; Política Social; Desarrollo Local y Economía Solidaria; Organización Comunitaria y Promoción Social; Redes Sociales y otros dispositivos de articulación de Actores; Instrumentos de Intervención Comunitaria; Trabajo Interdisciplinario del Abordaje Territorial. También se cursaban cuatro seminarios temáticos a elección del estudiante, las instancias de Autoaprendizajes con Tutorías, Seminarios de Integración y la elaboración del Trabajo Final Integrador, dicho trabajo debía sistematizar la práctica en servicio en el marco de la Especialización.

\section{Los trabajos finales integradores}

En el marco del presente artículo se toman como objeto de análisis los distintos Trabajos Finales Integradores que se encuentran en el repositorio institucional de la UNLA, y que fueron presentados entre los años 2009 -primeros trabajos de la carrera de posgrado- y 2011 -inclusive-, año en que se realizó el trabajo de campo en el CIC de Concordia. Estos corresponden a los que están con acceso completo en el repositorio institucional de la Especialización-UNLA (Mariani, 2009; La Barba, 2009; Gotti, 2010; Rizzo, 2010; Nestares, 2010; López Liatto, 2010; Alonso, 2011; Barés, 2011; Ramírez, 2011). Cada trabajo final pone énfasis en una temática específica que el profesional optó por profundizar, fruto de su experiencia de campo.

Por eso, es importante tomar la voz de estos actores en tanto son experiencias profesionales de distintas provincias de la Argentina y de distintas profesiones del campo de lo social, pues en su heterogeneidad es donde radica su riqueza ${ }^{14}$. En dichos trabajos se analizó específicamente cómo se desarrolló la experiencia de las Mesas de

\footnotetext{
${ }^{14}$ Mariani (2009) y Nestares (2010), provincia de Santa Fe, no especifican profesión; La Barba (2009), Misiones, licenciada en Comunicación Social; Rizzo (2010), Santa Fe, antropóloga; Gotti (2010), Santa Cruz, licenciada en Musicoterapia; López Liatto (2010), Tucumán, no especifica profesión; Alonso (2011), Tucumán, profesor en Juegos Teatrales. Barés (2011), Río Negro, comunicadora social; Ramírez (2011), Corrientes, asistente social.
} 
Gestión en cada localidad, con el objetivo de tener una visión más amplia de estos dispositivos.

Los especialistas como parte de la Universidad, y en vínculo con el MDSN, en su práctica cotidiana intentaron difundir entre los destinatarios de las políticas, los profesionales y los diferentes actores locales, los objetivos que perseguía la política de los $\mathrm{CIC}$, y realizaron acciones para fomentar la participación de la comunidad en la política del CIC y construir las mesas de gestión. A continuación, se desarrolla detalladamente la experiencia de cada uno.

Mariani (2009) establece que la articulación del CIC, el Municipio y la comunidad es "inexistente" (2009: 47) y en el sugestivo título La experiencia de la mesa de Gestión Local: ¿participación real? (2009: 66) se ocupa de reflexionar sobre la Mesa de Gestión. En su exposición narra que desde la Municipalidad no se realizó una convocatoria "masiva" para participar de la mesa, señalando que "los actores participantes en ese momento fueron, en su mayoría, personas empleadas de la comuna local, que estaban formando parte de alguna organización pero al mismo tiempo eran empleados de la comuna local" (2009: 67). La profesional afirma que la autoridad local "no fomenta la organización de la comunidad, sino que por el contrario trataron de socavarla, a fin de sostener una estructura verticalista y autoritaria de gobierno" (2009: 71).

La Barba (2009), por su parte, plantea que "al no haber en Santa Ana una mesa de gestión plural representativa de todos los sectores de la comunidad" (2009: 64), realizó su intervención articulando con instituciones representativas. Desde su profesión de base plantea que en la localidad donde le tocó intervenir, la comunicación en las políticas sociales era "centralizada y vertical" (2009: 49).

Gotti (2010), a su vez, refiere a la existencia de "modelos tradicionales de gestión con lógica verticalista que no facilitaban la participación interinstitucional ni de la comunidad en la estrategia de identificación y abordajes de sus problemas" (2010: 27); explicita que los CIC no tenían conformadas las Mesas de Gestión y "sus actividades no eran planificadas, ni discutidas por un dispositivo semejante" (2010: 27), su trabajo se orientó a revertir dicho proceso desde la capacitación vinculada a la forma de abordaje de las problemáticas sociales desde los CIC.

En su TFI, Ramírez (2011) plantea que el gobierno municipal no compartía los principios de los $\mathrm{CIC}$, por lo que "desde el inicio de la implementación del proyecto se fue tergiversando el espíritu de las acciones propuestas desde el MDS" (2011: 28). En la relación conflictiva entre el municipio y el MDSN, también se debatía el rol del especialista que se encontraba formando en los CIC, Ramírez lo expresa así: "Entendemos que poco se asemeja lo que esperan la UNLa ${ }^{15}$ y el MDS del especializando y lo que esperan los gobiernos locales del profesional que llega al

\footnotetext{
${ }^{15}$ Universidad Nacional de Lanús.
} 
Territorio" (2011: 40); concebido por estos como recurso del municipio para realizar actividades diferentes: "El gobierno local intentaba captar al especializando, en tanto recurso humano capacitado, para realizar tareas asistenciales, de control y de gestión de recursos, desconociendo por completo su potencial en la organización y promoción comunitaria y los objetivos propios de su proceso de formación en terreno" (ídem).

Respecto al tema que nos interesa en el presente artículo, Ramírez reflexiona: "En el CIC Santa Lucía, la 'Mesa de Gestión' se reunió solo en 6 oportunidades durante el período de 1 año, todas las veces que la Coordinadora convocaba a los miembros fue porque vendría algún referente de Nivel Central del MDS, de este espacio solo participaban funcionarios municipales y 'punteros políticos' de barrios lejanos al CIC. La coordinadora del CIC entendía que sobre ella recaía la responsabilidad de convocar a estas reuniones (de manera exclusiva) por lo cual elegía quienes serían los invitados" (2011: 57). En las reflexiones finales, la asistente social afirma: "un edificio administrado por una gestión municipal se vuelve un no lugar para todos aquellos ciudadanos que no son afines políticos a esa gestión" (2011: 93).

La experiencia de López Liatto (2010) plantea la conformación de la mesa de gestión, la cual se abocó a trabajar con jóvenes; según su propuesta, lo valioso de la experiencia fue la articulación interdisciplinaria, planteando como desafío la relación de la mesa con otros espacios, en sus palabras: "Fue difícil hacer valer este lugar frente a otros estamentos, sobre todo el municipal, pero con la fuerza de lo colectivo y con la necesidad de creer en otros proyectos posibles, se logró sostener la mesa de gestión como espacio donde pensamos y construimos entre todos. Cada uno aportó el saber necesario para abordar algunas problemáticas sentidas por los jóvenes" (2010: 67).

De la lectura y análisis de los distintos TFI, sobresale la relación conflictiva que se generó entre el MDSN, la Nación denominada por los diferentes actores y las autoridades de los distintos municipios, quienes en la mayoría de los casos intentaron eludir la convocatoria a la mesa de gestión, o realizarla de un modo parcial, ocupando el dispositivo con empleados estatales municipales (Mariani, 2009) o mesas que no eran plurales (La Barba, 2009).

Los principios de participación y democratización de las nuevas políticas sociales en los que se enmarcan las Mesas de Gestión y los CIC se chocaron con territorios en los que existía una "lógica verticalista" (Gotti, 2010), "piramidal" (Ramírez, 2011) o "centralizada" (La Barba; 2009). Es importante señalar que la existencia de las Mesas de Gestión era una condición sine qua non que solicitaba el MDSN para la aprobación del proyecto $\mathrm{CIC}$, y para las sucesivas políticas que se implementaban desde la Nación una vez construido el edificio. En espacios donde todavía los CIC estaban en proceso de construcción, las mesas de gestión no se pudieron constituir (Rizzo, 2010; Nestares, 2010; Alonso, 2011), ya que no había una construcción "ni material ni simbólica del CIC" (Rizzo, 2010:9); en el caso de Barés (2011), la comunicadora social se abocó a otra 
experiencia en marcha de una mesa de concertación, trabajando desde el Centro de Referencia del MDSN ${ }^{16}$.

\section{Reflexiones finales}

Si bien la Mesa de Gestión en el discurso de las nuevas políticas sociales es presentada con características que incluyen gran parte del ciclo de las políticas -planificación, ejecución, monitoreo y evaluación-, las mismas son predominantemente informativas y las decisiones de implementación de un plan, programa o proyecto en el marco de los $\mathrm{CIC}$ no es algo que se decide en una reunión de Mesa de Gestión, sino que es decidido centralizadamente desde el Ministerio de Desarrollo Social y los municipios, y en menor medida desde las provincias.

La Mesa de Gestión no tiene influencia en el rumbo de las diferentes situaciones cotidianas del $\mathrm{CIC}$, sino que es el coordinador del $\mathrm{CIC}$, o este junto a los referentes comunitarios. Entonces, las reuniones de la Mesa de Gestión son espacios informativos y de coordinación de actividades y políticas que ya se encuentran decididas por las autoridades y los profesionales del Ministerio.

Tampoco son espacios abiertos, plurales a donde se acercan los vecinos a participar; ya que como se remarca en la experiencia de Concordia, son los representantes de la comunidad y los referentes comunitarios los que tienen voz y voto en las mismas.

Dichos dispositivos fueron un requisito en el marco de los $\mathrm{CIC}$ promovidos por el Estado nacional, el cual tuvo múltiples resistencias y resignificaciones en el marco de los distintos territorios en los que fueron implementados, las situaciones que sobresalieron fueron dos: la no realización de las mesas de gestión o la construcción de mesas sin pluralidad de voces.

Es por ello que, tomando la experiencia de Concordia y las narradas por los diferentes especialistas en sus Trabajos Finales Integradores, se puede afirmar que la existencia de estos dispositivos de participación promovidos por el Estado en el marco de políticas de desarrollo social no necesariamente conducen a la participación de la comunidad y la democratización de dichas políticas.

El objetivo que persiguió el presente texto fue reflexionar sobre el dispositivo de participación de las Mesas de Gestión, y aportar al debate de las políticas de desarrollo social y la participación de los destinatarios en las mismas.

\section{Bibliografía}

Alonso, E. B. (2011). El fortalecimiento de la identidad cultural como una apuesta para el desarrollo Sustentable [en Línea]. Universidad Nacional de Lanús. Departamento

\footnotetext{
${ }^{16}$ Los Centros de Referencia son delegaciones del Ministerio de Desarrollo Social en cada una de las provincias de la Argentina. Ver Bazzalo (2011).
} 
de Salud Comunitaria. Disponible en: http://www.repositoriojmr.unla.edu.ar/descarga/TFI/EsAIP/031949_Alonso.pdf Arroyo, D. (2004). Prólogo. El rol de los Consejos Consultivos en el marco del Desarrollo Local. En González Bombal, I. Fortaleciendo la relación Estado-Sociedad Civil para el Desarrollo Local. Buenos Aires: Libros del Zorzal-CEDES-CENOC-Universidad Nacional de General Sarmiento, pp. 13-19.

Arroyo, D. (2006). Desarrollo y políticas públicas. Nuevos desafíos para el Estado y la sociedad civil. En García Delgado, D. y Nosetto, L. El desarrollo en un contexto posneoliberal. Hacia una sociedad para todos. Buenos Aires: Ediciones CICCUS, pp. 195-217.

Barés, A. (2011). Presentes urgentes: jóvenes construyendo presentes en contextos atravesados por los desafíos de la interculturalidad y las des (igualdades) [en Línea]. Universidad Nacional de Lanús. Departamento de Salud Comunitaria. Disponible en: http://www.repositoriojmr.unla.edu.ar/descarga/TFI/EsAIP/031952_Barés.pdf

Bazzalo, A. (octubre de 2011). Discursos habilitantes y nuevos escenarios en las políticas sociales. Matrices, factores y ámbitos de reproducción: El caso de los Centros de Referencia del Ministerio de Desarrollo Social de la Nación. Revista Debate Público, 2, pp. 101-110.

Bourdieu, P. (1997). Espíritus de Estado. Génesis y estructura del campo burocrático. En Bourdieu, P. Razones Prácticas. Sobre la teorías de la acción. Barcelona: Anagrama, pp. 91-138.

Bourdieu, P. y Wacquant, L. (2008). Una invitación a la sociología reflexiva. Buenos Aires: Siglo XXI.

Carrasco, M. (2012). Políticas Sociales en el Congreso de la Nación Argentina 20022009. Tesis de Maestría FLACSO, Buenos Aires, FLACSO Andes. Disponible en: http://repositorio.flacsoandes.edu.ec/handle/10469/3770\#.Vx6INNThDDc

Castronovo, R. y García, A. (2013). Reconstrucción de una experiencia. Carrera de Especialización en Abordaje Integral de las Problemáticas Sociales en el Ámbito Comunitario. Remedios de Escalada: Ediciones de la UNLa.

Corrigan, P. (2002). La formación del estado. En Joseph, G. y Nugent, D. Aspectos cotidianos de la formación del estado. México: Era, pp. 25-27.

Corrigan, P. y Sayer, D. (2007). El gran arco: la formación del estado inglés como revolución cultural. En Lagos, M. y Calla, P. Antropología del Estado. Dominación y prácticas contestatarias en América Latina. Cuaderno de Futuro 23. Bolivia: INDH/PNUD, pp. 39-59.

De Piero, S. (2015). Los Centros Integradores Comunitarios: nuevos modos de territorialidad del Estado Nación. En Grandinetti, R.; Beretta, D.; Schweinheim, G. y 
Rey, M. Retos e innovaciones de la administración pública para el desarrollo democrático en el Siglo XX. Rosario: Libro Digital PDF, pp. 104-115.

Del Prado, L. (2009). Políticas sociales y democracia. Reflexiones sobre la Mesa de Gestión de un Centro Integrador Comunitario. Revista Regional de Trabajo Social, pp. 62-67.

Del Prado, L. (2015). Alicia Kichner: la ideóloga de las nuevas políticas sociales en Argentina. Más Poder Local, pp. 18-20.

Golbert, L. (2004). ¿Derecho a la inclusión o paz social ? Plan Jefas y Jefes de Hogar Desocupados. Santiago de Chile: CEPAL. ONU.

Gotti, S. V. (2010). Mesa de gestión, una oportunidad de reparación social [en Línea]. Universidad Nacional de Lanús. Departamento de Salud Comunitaria. Disponible en: http://www.repositoriojmr.unla.edu.ar/descarga/TFI/EsAIP/032234_Gotti.pdf

Gutiérrez, A. (1994). Pierre Bourdieu: las prácticas sociales. Buenos Aires: Centro Editor de América Latina.

Kirchner, A. (2007a). La Bisagra. Memoria/Verdad/Justicia/Organización Social. Buenos Aires: Ministerio de Desarrollo Social de la Nación.

Kirchner, A. (2007b). CIC. Centro Integrador Comunitario. Una oportunidad para la organización. Cuadernillo $N^{\circ}$ 1. Buenos Aires: Consejo Nacional de Coordinación de Políticas Sociales. Presidencia de la Nación.

Kirchner, A. (2008). Los Centros Integradores Comunitarios. Sentido de la Política Social=El Umbral del Cambio. En Kliksberg, B. Pensamiento Social Estratégico. Una nueva mirada a los desafíos sociales de América Latina. Buenos Aires: Siglo XXI, pp. 107-111.

Kirchner, A. (2010a). Políticas Sociales del Bicentenario. Un Modelo Nacional y Popular (Vol. 1). Buenos Aires: Ministerio de Desarrollo Social de la Nación.

Kirchner, A. (2010b). Políticas Sociales del Bicentenario. Un Modelo Nacional y Popular. Glosario. Definiciones y Contexto (Vol. 2). Buenos Aires: Ministerio de Desarrollo Social de la Nación.

Kirchner, A. y Vessvessian, P. (2001). En Busca del Ordenador Social. Trabajo: Agenda no resuelta. Buenos Aires: Espacio.

La Barba, F. V. (2009). Experiencias de educación y comunicación popular en escuelas públicas de Santa Ana [en Línea]. Universidad Nacional de Lanús. Departamento de Salud Comunitaria. Disponible en: http://www.repositoriojmr.unla.edu.ar/descarga/TFI/EsAIP/0228810_LaBarba.pdf

López Liatto, J. M. (2010). Una posible estrategia de abordaje integral de problemáticas de jóvenes de zonas rurales [en Línea]. Universidad Nacional de Lanús. Departamento de Salud Comunitaria. Disponible en: http://www.repositoriojmr.unla.edu.ar/descarga/TFI/EsAIP/031953_Lopez_Liatto.p df 
Mariani, V. (2009). La participación social en la localidad de Sauce Viejo, un proceso a construir desde la comunidad [en Línea]. Universidad Nacional de Lanús. Departamento de Salud Comunitaria. Disponible en: http://www.repositoriojmr.unla.edu.ar/descarga/TFI/EsAIP/033717_Mariani.pdf

Martínez, S. (2015). Cambio de paradigma en políticas sociales. En C. N. MOSTUNESCO, Radiografía de las políticas sociales del siglo XXI. Las miradas populares. s.d.: s.d., pp. 241-245

Matus, C. (1972). Estrategia y plan. Chile: Siglo XXI.

Meroni, J. (2014). Trabajadores Sociales en el territorio: El Estado y las Políticas Sociales presentes en el ámbito barrial. Revista Debate Público, 8, pp. 111-121.

Mingo de Bevilacqua, G.; Sarrot, E.; Bitar, M.; González Alarcón, A.; Sione, C. y Romero, G. (2006). Dimensiones de la pobreza en Paraná y Concordia. Un estudio comparativo. Ciencia, Docencia y Tecnología, 33, pp. 67-110.

Mingo, G.; Sarrot, E.; González Alarcón, A.; Zapolsky, S.; Bitar, M., Sione, C. y otros. (2006). Pobreza urbana: discursos y sujetos. Buenos Aires: Espacio.

Ministerio de Desarrollo Social de la Nación Argentina. (2015). Políticas Públicas con Impacto Social. s.d.: s.d.

Morresi, S. (2007). ¿Más allá del neoliberalismo? Estado y neoliberalismo en los años noventa. En Rinesi, E.; Nardacchione, G. y Vommaro, G. Las lentes de Victor Hugo. Transformaciones políticas y desafíos teóricos en la Argentina reciente. Buenos Aires: UNGS. Prometeo, pp. 117-150.

MOST-UNESCO (2015) Radiografía de las políticas sociales del siglo XXI. Las miradas populares. s.d.: s.d.

Nestares, G. C. (2010). Crece desde el pie... sistematización de una experiencia de economía social de la ciudad de Granadero Baigorria, Santa Fe [en Línea]. Universidad Nacional de Lanús. Departamento de Salud Comunitaria. Disponible en: http://www.repositoriojmr.unla.edu.ar/descarga/TFI/EsAIP/032200_Nestares.pdf

Oszlak, O. (2001). El Estado transversal. Encrucijadas, 6, pp. 30-41.

País Andrade, M. (2010). Espacios fronterizos e identidades. Tensiones y estrategias político-culturales en la ciudad de Concordia. Revista RUNA. Archivos para la Ciencia del Hombre, pp. 175-190.

Ramírez, S. (2011). Reflexiones en torno a la construcción del rol del especializando en el territorio: discursos y prácticas en la implementación del proyecto $\mathrm{CIC}$ en la localidad de Santa Lucía, Provincia de Corrientes, durante los años 2006 y 2007 [en Línea]. Universidad Nacional de Lanús. Departamento de Salud Comunitaria. Disponible en: http://www.repositoriojmr.unla.edu.ar/descarga/TFI/EsAIP/032233_Ramirez.pdf 
Repetto, F. y Chudnovsky, M. (2009). Las políticas sociales en la Argentina reciente. En Quiroga, Y.; Canzani, A. y Ensignia, J. Consenso progresista. Las políticas sociales de los gobiernos progresistas del Cono Sur. Fundación Friedrich Ebert, pp. 15-45.

Reta, M.; Rossi, G. y Toler, S. M. (2008). Evaluación de las políticas alimentarias. Consideraciones metodológicas a partir del caso de la ciudad de Concordia. Concepción del Uruguay: EDUNER.

Rizzo, L. (2010). Género y participación comunitaria en contextos de interculturalidad: relato de una experiencia de trabajo con distintas mujeres en una comunidad de Santo Tomé, Santa Fe [en Línea]. Universidad Nacional de Lanús. Departamento de Salud Comunitaria. Disponible en: http://www.repositoriojmr.unla.edu.ar/descarga/TFI/EsAIP/031938_Rizzo.pdf

Robirosa, M.; Cardarelli, G. y La Palma, A. (1990). Turbulencia y Planificación Social. Lineamientos metodológicos de gestión de proyectos sociales desde el Estado. Buenos Aires: Siglo XXI. UNICEF.

Vilas, C. (2011). De la focalización a la integralidad. Cambio de paradigma en las políticas contra la pobreza y la desigualdad. En Vilas, C. Después del Neoliberalismo: Estado y procesos políticos en América Latina. Remedios de Escalada: Universidad Nacional de Lanús, pp. 147-164. 